\title{
Systemic Management of Dysthyroid Ophthalmopathy
}

\author{
PETER FELLS
}

London

\begin{abstract}
Summary
The best systemic treatment of dysthyroid ophthalmopathy remains high dosage Prednisolone. Other methods of immunosuppression such as Azathioprine and Cyclosporin A have proved disappointing, as has plasmapheresis. Other localised means may be used and these are radiotherapy to the retrobulbar tissues or orbital decompression. These methods may be used in addition to or instead of high dose Prednisolone but some surgeons prefer to try Prednisolone as a first measure.
\end{abstract}

Thyroid eye disease is a difficult condition to manage because it is a poorly defined yet generally recognised clinical condition with multiple symptoms, incompletely understood pathological processes and a prolonged and variable natural history. Under such circumstances it is not surprising that many therapies have been tried, but randomised, prospective trials are almost impossible to carry out and ever fallible clinical impressions abound. In practice it is by attention to detail in the individual patient, and the use of local and systemic drugs together with carefully timed surgical intervention that worthwhile results may be obtained. This paper is concerned with systemic treatment but inevitably the other forms will be mentioned where appropriate.

The patient may be concerned by loss of vision, from corneal exposure or optic nerve compression, double vision from ocular muscle tethering, the appearance of proptosis, lid retraction, conjunctival injection or any combination of these features. Active, congestive thyroid eye disease tends to remit within three years and only 10 per cent of patients develop serious long term ocular problems. Lid retraction improves in 50 per cent, but proptosis persists in 70 per cent although chemosis and conjunctival injection decrease with the passing years. Diplopia may be transient, but in 50 per cent of patients it stays. Vision is acutely threatened in only $2-7$ per cent of dysthyroid eye patients. ${ }^{1}$

Between 30 per cent and 50 per cent of hyperthyroid patients have ophthalmophathy. Treatment of the hyperthyroidism improves the lid retraction in most cases. The options for control of thyrotoxicosis are oral carbimazole, surgical thyroidectomy and radioactive iodine therapy. Once adequate remission of thyrotoxicosis has been achieved these latter two methods are almost invariably followed by hypothyroidism after a period of years.

Catz and colleagues ${ }^{2}$ produced complete thyroid ablation by either total thyroidectomy followed by radioiodine, or by large doses of ${ }^{131} \mathrm{I}$ in attempts to prevent and treat ophthalmopathy. This was on the basis that the antigen that elicits the antibody response that causes the ophthalmopathy is produced in the thyroid gland. Unfortunately thyroid ablation inevitably increases the release of thyroglobulin which may be largely responsible for the ophthalmopathy. No other workers have been able to reproduce the excellent results reported by these authors. 
Carbimazole tablets may be given in two ways, either a constant daily dose until a predetermined total has been taken with concurrent ingestion of thyroxine tablets to prevent any temporary hypothyroidism, or the carbimazole is adjusted every two or three months to give optimal control of the hyperthyroidism as judged by clinical signs and circulating thyroid hormones. Most clinicians agree that slipping into hypothyroidism may exacerbate the ophthalmopathy. Recurrent hyperthyroidism has a bad prognosis for ocular involvement too. Carbimazole is considered to have an immunosuppressive effect in addition to inhibiting thyroid hormone synthesis and this may be on the ophthalmopathy as well as on the thyroid gland.

At the tissue level in thyroid ophthalmopathy the pathological changes are of interstitial oedema, penetrating between individual muscle fibres and fat cells with infiltration by lymphocytes, plasma cells, macrophages and mast cells. ${ }^{3}$ Fibroblasts, in response to stimulation by increasing lymphocytes, enlarge and proliferate and themselves produce hyaluronic acid, glycosaminoglycans and collagen. ${ }^{4}$ With time the inflammation becomes chronic with fibrotic scarring and fatty infiltration of the muscles restricting ocular rotations.

Systemic therapy can only hope to be of value in the early infiltrative stages before permanent scarring occurs. Thiazide diuretics, such as Navidrex-K containing 250 micrograms of cyclopenthiazide, are given as one or two tablets at night to reduce the morning accumulation of oedema fluid in the face and orbit.

Various pathogenetic interactions that could lead to the observed tissue changes have been proposed, including immunological reactions between antibodies and orbital and thyroid antigens, immune complexes with ocular muscle membranes and other possible reactions of autoimmunity which are beyond the scope of this paper. Initially corticosteroids and, later, other immunosuppressants have been used to try to prevent these tissue reactions occurring. ${ }^{6}$ It is now established that high oral doses of prednisolone $80-100 \mathrm{mg}$ a day can reduce the acute, congestive stage of dysthyroid proptosis and also relieve optic nerve compression at the orbital apex usually within 48 hours. However, to maintain remission the dose must be maintained at high levels for weeks or months with only a gradual reduction. Many patients find that the frequent side effects of such therapy are not acceptable. These include diabetes, systemic hypertension, peptic ulceration, osteoporosis, psychosis, bruising, hirsutism, cataracts etc. Very recently an intravenous bolus of a high concentration of methylprednisolone has been used in an attempt to reduce these side effects but I have no personal experience of this method. Steroids have minimal effect on thyroid ophthalmopathy that has been established for longer than a year. This means that steroids have little if any effect on reduced ocular movements or lid retraction.

Other immunosuppressants have been tried, in particular azathioprine, either alone or with prednisolone, in an attempt to keep the dose of prednisolone as low as possible. Maintenance prednisolone dose should not be more than $10 \mathrm{mg}$ per day. Winand and Mahieu $^{6}$ claimed that hyperthyroid patients with a positive leucocyte migration test were at risk of developing ophthalmopathy. All their patients were treated both for hyperthyroidism and with azathioprine $1.5 \mathrm{mg} / \mathrm{kg}$ / day and none developed severe ophthalmopathy. Neither of these diagnostic and therapeutic claims has been substantiated by other workers.

Because of the abnormal circulating immunoglobulins ( $\operatorname{IgG}$ ) in thyroid ophthalmopathy and the fact that plasmapheresis is effective in other conditions (e.g. hyperviscosity syndrome, Goodpasture's syndrome and myasthenia gravis) where specific circulating IgGs mediate the injury, Dandona and colleagues tried plasmapheresis in thyroid ophthalmopathy. ${ }^{7}$ The initial success was followed by a rebound recurrence over the next two weeks. Further plasmapheresis was used but simultaneously prednisolone $80 \mathrm{mg}$ per day and azathioprine $125 \mathrm{mg}$ /day were given. Later workers could not confirm any significant favourable results with plasmapheresis alone. ${ }^{9}$

A new immunosuppressant, cyclosporin A, is a potent, specific suppressant of $T$ cell func- 
tion but in addition it inhibits the $B$ cell humoral response to $\mathrm{T}$ cell dependent antigens. A personal, combined study with the endocrinologists at St. Bartholomew's Hospital failed to show any significant effect in three patients with moderate degrees of dysthyroid ophthalmopathy and could not confirm the improved ocular rotations reported by Weetman et al. ${ }^{9}$

This survey of systemic therapy for thyroid ophthalmopathy has not shown any single best treatment. Other more localised methods used for sight threatening dysthyroid diseases include orbital decompression ${ }^{10}$ and radiation therapy. ${ }^{11}$ Supervoltage radiation with the linear accelerator aiming a posteriorly angled lateral beam to pass behind the lens of the eye and anterior to the pituitary body has been effective, particularly where the orbital disease is of less than one year's duration.

Whatever the immunological processes in the thyroid and orbit may be, any therapy to be effective must be delivered before permanent tissue changes have occurred. To date oral steroids are still the best treatment available, possibly combined with retrobulbar radiotherapy. Surgery for the chronic state of ocular muscle fibrosis continues to have an important role in reducing diplopia and helping these unfortunate patients. ${ }^{12}$
References

'Hales IB and Rundle FF: Ocular changes in Graves' disease. Quart J Med 1960; 29: 113-26.

${ }^{2}$ Catz B and Perzik SL: Total thyroidectomy in the management of thyrotoxic and euthyroid Graves' disease. Am J Surg 1969; 118: 434-9.

${ }^{3}$ Riley FC: Orbital pathology in Graves' disease. Mayo Clinic Proc 1972; 47: 975-9.

+ Campbell RJ: Pathology of Graves' ophthalmopathy in The Eye and Orbit in Thyroid Disease, ed. by Gorman et al, New York. Raven Press, 1984: 25-31.

${ }^{5}$ Werner SC: Prednisone in emergency treatment of malignant exophthalmos. Lancet 1966; 1: 1004-7.

${ }^{6}$ Winand $\mathrm{R}$ and Mahieu P: Prevention of malignant exophthalmos after treatment of thyrotoxicosis. Lancet 1973; 1: 1196.

${ }^{7}$ Dandona P, Marshall NJ, Bidey SP, Nathan A, Havard CWH: Successful treatment of exophthalmos and pretibial myxoedema with plasmapheresis. Br Med J 1979; 1: 374-6.

${ }^{8}$ Kelly W, Longson D, Smithard D, Fawcitt R, Wensley R, Noble J, Keeley J: An evaluation of plasma exchange for Graves' ophthalmopathy. Clin Endocrinol (Oxf) 1983; 18: 485-93.

${ }^{9}$ Weetman AP, McGregor AM, Ludgate M, Beck L, Mills PV, Lazarus JH, Hall R: Cyclosporin improves Graves' ophthalmopathy. Lancet 1983; 2: 486-489.

10 Fells P: Orbital decompression for severe dysthyroid eye disease. Br J Ophthalmol 1987; 71: 107-11.

"Pinchera A, Bartalena L, Chiovato L, Marcocci C: Radiotherapy of Graves' ophthalmopathy in The Eye and Orbit in Thyroid Disease ed. by Gorman et al, New York. Raven Press, 1984: 301-16.

12 Fells $\mathrm{P}$ and McCarry B: Diplopia in Thyroid Eye Disease. Trans ophthalmol Soc UK 1986; 105: 413-23. 